Special issue of the 3rd International Conference on Computational and Experimental Science and Engineering (ICCESEN 2016)

\title{
Application of Data Communication to the Detection and Correction of Power System Faults
}

\author{
A. Recioui*, M. Lograda And K. Chettah \\ Laboratory Signals and Systems, Institute of Electrical Engineering and Electronics, \\ University M'hamed Bougara of Boumerdes, Av. de l'Indépendance, 35000 Boumerdes, Algeria

\begin{abstract}
Data communication is an important part in modern industrial processes and is necessary in automation, monitoring, and protection purposes. This paper aims at designing a real time communication between two computers for interchanging the state of a three phase circuit to emulate a transmission line protection system in power substation. The circuit designed in Proteus ISIS is based on "symmetrical component theory" and the algorithm for exchanging data between two computers is built using TCP/IP protocol in LABVIEW environment. The application consists of monitoring and controlling three phase lines by sending the data collected to one of the computers and sending the commands back to trip relays if any fault is detected. The purpose is to prevent the propagation of this fault and to connect other sources to maintain the flow of power to the load.
\end{abstract}

DOI: 10.12693/APhysPolA.132.819

PACS/topics: power system faults, data communication, fault detection, symmetrical components, LABVIEW

\section{Introduction}

Faults in power system usually occur due to the deterioration of insulating materials, natural phenomena, or human errors. These faults may be either symmetrical involving all the three phases or asymmetrical where only one or two phases may be involved. These faults are either short circuited to ground or between line conductors. Balanced three phase faults can be analyzed using an equivalent single phase circuit, however in case of unbalanced faults it is quite complex when taking only one phase into consideration. The most common method to reduce the complexity of computations is to use "symmetrical components theory" [1, 2].

Engineers in power system are basically focusing on the behavior of the whole system when encountering any fault or abnormal deviations. This is called the monitoring of power system. To perform such operations it is extremely evident that manipulations on nominal and measured data are going to be done. Obviously it is unbelievable to say that one can monitor a system that lies over hundreds and hundreds of kilometers without the intervention of something else. In fact, a simple concept can clarify and solve such a riddle, it is to gather information and data and send them from different places to one local place where all these operations of processing, comparing, adjusting and controlling can be accomplished, such a process is called telecommunication [3-12].

Now, that this outcome comes to solve the problem of long distances, facing a lot of sub-problems will not be unavoidable such as data reliability, fastness, and cost efficiency. That is why in developed countries a special field has been created for concentrating studies on how it will

*corresponding author; e-mail: recmad2006@yahoo.fr be possible to bring the communication principles into the power sector for solving prospective problems $[4,13]$.

Nobody can talk about communication without underlining the protocol used for that communication. Ensuring the right and proper exchange of data is one of the roles of the protocol chosen for that. Mainly, the protocol is picked up depending on some outlined criteria. One seeks to exchange data in fast manner regardless the reliability of some data whereas others claim to have the whole information content $[16,17]$.

In this work, the link between the communication and power is investigated starting from fault creation, passing by fault detection ending up by the right control to cure the detected fault.

\section{System description}

In this section, we are going to build the communication part, first; between the Proteus software and LABVIEW using serial communication RS232; and then between the first computer where the TCP client is programmed and the second computer acting as TCP server [14, 15].

The main goal of this work is to design an application demonstrating how communication is being used in power systems. The proposed application is to use data exchange between two PCs where the first one will act as substation carrying on dynamic data gathered from the fault detection circuit, to cast a general view about the state of transmission line and the second computer will be the control center $[18,19]$. A client/server communication using TCP/IP protocol is programmed in the two PCs using LABVIEW software.

\subsection{ISIS environment}

Mainly, this part will interface Proteus with LABVIEW; data are sent serially back and forth between 
these two software packages. These data might be either the state of the LEDs or the commands received from the server to the client to trip a relay depending on the type of fault.

\subsection{Serial communication RS232}

Serial communication is basically the transmission or reception of data one bit at a time. It can be used for short distance data transmission only. In this application it is used to exchange data between Proteus and LABVIEW. In Proteus this is done by using the combination of any microcontroller with serial port COMPIM (Fig. 1) where the microcontroller has to be programmed in order to the serial port to carry on the desired data to send and to receive $[20]$.

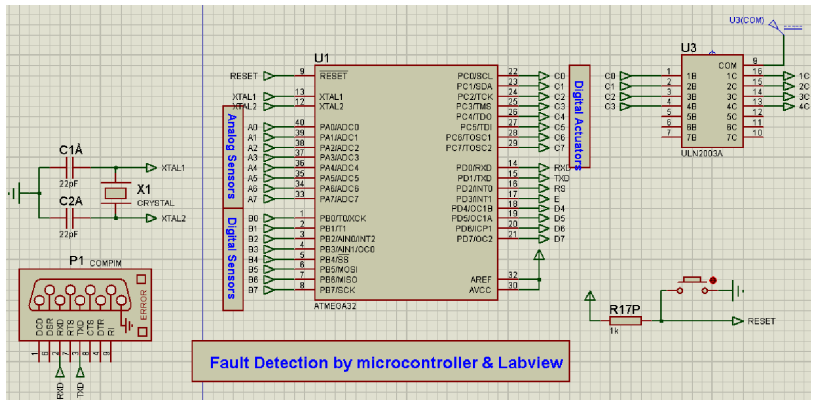

Fig. 1. The diagram of serial interface with LabVIEW.

This Atmega32 powerful microcontroller is programmed to achieve the serial communication between the Proteus and LABVIEW. Two ports of this microcontroller are used as inputs namely PORT_A and PORT_B, where PORT_A (8-pins) are analog used to read analog signals and PORT B (8-pins) are digital (either high or low depending on the fault in the circuit). Another port which is PORT_C (8-pins) is used as digital outputs to actuate the state of the relays.

The main function of the program developed in C language using Micro $\mathrm{C}$ software, is composed of two parts:

- Part1: reception of data from the computer.

- Part2: send of data to the computer.

\section{Simulations results and discussions}

To demonstrate the process of the application, fault detection has been simulated. The chosen fault is the line to ground fault. This will be detected in ISIS circuit and fed to the TCP client and then to TCP server.

When the circuit works under normal condition (no fault), the NO_FAULT_LED of TCP client and server front panelswill be $\mathrm{ON}^{-}$to indicate that everything is working properly. It clearly indicates that no fault happened in the three phase lines.

Now, the third push button in the circuit is activated to short circuit one line to ground. The LG FAULT LED is consequently turned ON. The microcontroller will sense this change in PORT B and send serially data to the first computer a frame containing a total of " 2 ". This means that the second LED indicator in TCP CLIENT front panel will glow. Similarly, the same frame will be sent from TCP CLIENT to TCP SERVER in the second computer via Ethernet cable and decoded to make the LG indicator turn ON.

In NO_FAULT condition, the three push buttons are all open and the three loads shown in Fig. 2 are all having power from the generator. In LG_FAULT only, two loads are $\mathrm{ON}$ whereas LOAD_3 is not receiving power since the third push button is pressed and hence the third line from the generator is short circuited to ground.

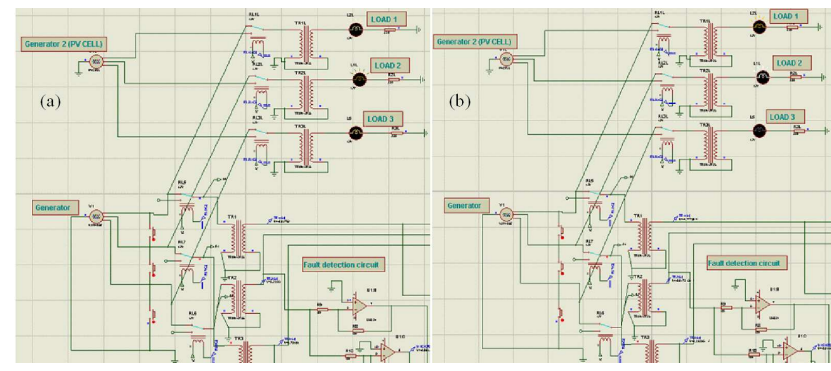

Fig. 2. State of relays and loads during (a) NO FAULT, (b) LG_FAULT in ISIS.

Once the frame of data is received in the control

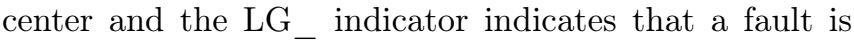
happening, a right control must be taken. Here, the TCP SERVER will send two distinct commands; the first one is for tripping OFF the relay of the generator to isolate the circuit and the second command will trip ON the relay of PV_CELL_GENERATOR to maintain the circulation of power to the LOADS.

When the commands are received by the microcontroller, it will output two signals through PORT_C. These signals will be amplified by the ULN2003A to be able to trip the relays and hence make the PV_CELL the alternative generator to feed the LOADs (Fig. 3).

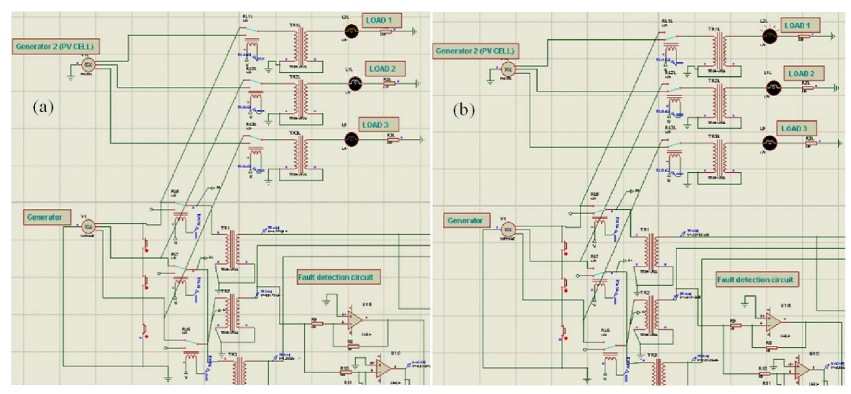

Fig. 3. State of relays and loads: (a) before receiving the commands, (b) after receiving the commands.

This application can also display the three line voltages of the GENERATOR via analog port (PORT A). The only disadvantage is that the microcontroller converts the 
analog inputs into unsigned digital values from 0 to 255 , which means that the graph will display the minimum analog input as zero and the maximum as 255 .

\section{Conclusion}

A fault detection approach in three-phase circuit has been presented based on "symmetrical component theory". Proteus software was used for this simulation. Once the fault is detected it has to be sent from the substation to the control center. In this paper, one computer is used as substation gathering information about the state of the fault detection circuit and another computer is used as control center that receives the data and processes the right control of the exchange of data between these two computers is done via Ethernet TCP/IP. The simulations can be considered as an illustration of how data communication is used for power system fault detection and correction.

\section{References}

[1] K. Djaballah Boudjebiba, M. Boussentoh, Final Year Project Report, Institute of Electrical and Electronic Engineering, Boumerdes 2012.

[2] O. Boukhenane, H.E. Debbah, Final Year Project Report, Institute of Electrical and Electronic Engineering, Boumerdes 2012.

[3] S. Mohagheghi, J. Stoupis, Z. Wang, Communication Protocols and Networks for Power Systems - Current Status and Future Trends, IEEE Explore, 2009.
[4] OSI reference model (Open Systems Interconnection), TechTarget.

[5] A. Stelios, Networking Basics: TCP, UDP, TCP/IP and OSI Model.

[6] The TCP/IP Guide, TCP Characteristics: How TCP Does What It Does.

[7] The TCP/IP protocol suite, Unicen.

[8] TCP/IP Tutorial and Technical Overview December 2006.

[9] Modbus Protocol Reference Guide PI-MBUS-300 Rev. J, Modicon, North Andover 1996.

[10] G. Thomas, Extension, Control Network 9, 4 (2008).

[11] R. Hair, Modbus interface tutorial, 2009.

[12] MODBUS over serial line specification and implementation guide, V.1.

[13] J.D. Glover, S. Sarma, T. Overbye, Power System Analysis and Design, 4th ed., Cengage 2007.

[14] Functions and VI Reference Manual, National Instruments Corporation, 1998.

[15] LABVIEW Demonstration Guide, National Instruments Corporation, 1996.

[16] A. Özdemir, Z. Erdem, I. Usuman, Acta Phys. Pol. A 130, 228 (2016)

[17] T. Aydogan, I. Ozcelik, I. Erturk, H. Ekiz, Acta Phys. Pol. A 130, 412 (2016).

[18] P. Stefanov, A. Savić, G. Dobrić, Acta Phys. Pol. A 128, B-138 (2016).

[19] M.J. Pazdanowski, Acta Phys. Pol. A 128, B-213 (2016).

[20] Y. Sarikaya, H. Apaydin, Ş. Kıtış, Acta Phys. Pol. A 128, B-211 (2016) 